\title{
Mitochondrial DNA 7908-8816 region mutations in maternally inherited essential hypertensive subjects in China
}

Ye Zhu ${ }^{1,2}$, Xiang $\mathrm{Gu}^{1,2^{*}}$ and Chao Xu ${ }^{3}$

\begin{abstract}
Background: Nuclear genes or family-based mitochondrial screening have been the focus of genetic studies into essential hypertension. Studies into the role of mitochondria in sporadic Chinese hypertensives are lacking. The objective of the study was to explore the relationship between mitochondrial DNA (mtDNA) variations and the development of maternally inherited essential hypertension (MIEH) in China.
\end{abstract}

Methods: Yangzhou residents who were outpatients or in-patients at the Department of Cardiology in Northern Jiangsu People's Hospital (Jiangsu, China) from June 2009 to June 2015 were recruited in a 1:1 case control study of 600 gender-matched Chinese MIEH subjects and controls. Genomic DNA was isolated from whole blood cells. The most likely sites for hypertension were screened using oligodeoxynucleotides at positions 7908-8816, purified and subsequently analyzed by direct sequencing according to the revised consensus Cambridge sequence. The frequency, density, type and conservative evolution of mtDNA variations were comprehensively analyzed.

Results: We found a statistical difference between the two groups for body mass index, waist circumference, abdominal circumference, triglyceride, low-density lipoprotein cholesterol, fasting blood glucose, uric acid, creatinine and blood urea nitrogen $(P<0.05)$. More amino-acid changes and RNA variants were found in MIEH subjects than the controls $(P<0.01)$. The detection system simultaneously identified 40 different heteroplasmic or homoplasmic mutations in 4 genes: COXII, tRNA ${ }^{\text {Lys }}$, ATP8 and ATP 6 . The mtDNA variations were mainly distributed in regions of ATP6 binding sites, and the site of highest mutation frequency was $\mathrm{m}$. 8414C $>$ T. Three changes in single bases (C8414T in ATP8, A8701G in ATP6 and G8584A in ATP6) were significantly different in the MIEH patients and the controls $(P<0.001)$. The m.8273_8281del mutation was identified from 59 MIEH patients.

Conclusions: Our results indicate that novel mtDNA mutations may be involved in the pathological process of MIEH, and mitochondrial genetic characteristics were identified in MIEH individuals.

Keywords: Mitochondria, DNA, Mutation, Essential hypertension, Maternal inheritance

\section{Background}

Essential hypertension (EH) is a common chronic disease which is becoming an urgent public health issue worldwide, accounting for 9.4 million deaths each year [1]. EH is characterized by an elevation in arterial pressure and is a major risk factor for many common causes of morbidity and mortality including myocardial

\footnotetext{
* Correspondence: guxiang@yzu.edu.cn

${ }^{1}$ Clinical Medical College, Yangzhou University, Yangzhou 225001, Jiangsu, China

${ }^{2}$ Department of Cardiology, Northern Jiangsu People's Hospital, Yangzhou 225001, Jiangsu, China

Full list of author information is available at the end of the article
}

infarction, congestive heart failure, stroke and kidney failure in many segments of the population [2]. EH results from the interaction between environmental and inherited risk factors, which can be caused by singlegene or multifactorial conditions.

A family history of hypertension means that individuals are more likely to suffer hypertension [3, 4]. Maternally inherited essential hypertension (MIEH) is EH that shows a pattern of maternal inheritance and is occasionally observed in the clinic [5]. mtDNA can lead to mitochondrial diseases that are exclusively transmitted from the mother. mtDNA mutations have been identified in

(C) The Author(s). 2018 Open Access This article is distributed under the terms of the Creative Commons Attribution 4.0 International License (http://creativecommons.org/licenses/by/4.0/), which permits unrestricted use, distribution, and 
some pathogenic diseases such as myoclonic epilepsy, lactic acidosis, mitochondrial myopathy, encephalopathy, stroke-like episodes, and ragged-red fibers or maternally inherited diabetes [6]. Accordingly, mutations in mtDNA have also been reported in MIEH [7].

Mitochondria have an inefficient DNA repair and protection system in comparison to that of nuclear DNA [8]. All the homoplasmic mtDNA mutations that have been identified as being related to MIEH have caused functional disorders. The m.4435A $>\mathrm{G}$ mutation that is located immediately at the 3'end of the anticodon. This location corresponds to position 37 of tRNAMet affecting codon recognition, structural formation, and stabilization of functional tRNAs [9]. The m.4263A $>\mathrm{G}$ mutation reduces the efficiency of the tRNAIle precursor 5 '-end cleavage that is catalyzed by RNase P because it is located at the processing site for the tRNAIle 5 '-end precursor [10]. The result of these mutations is abnormal mitochondrial respiration that causes oxidative stress, this uncouples the oxidative pathways for ATP synthesis, and leads to cellular energetic processes failing [11].

To date, the roles of somatic mtDNA mutations in MIEH are still poorly understood. The development of blood pressure and this increases with many factors that include the mtDNA mutation/background, nuclear genes and environmental factors [12]. There is some suggestion that gene variations are associated with hypertension; but different results have been seen in different populations [13]. The mitochondrial genome accounts for $\sim 5 \%$ of the heritability of blood pressure and these increases to $\sim 35 \%$ for hypertensive pedigrees $[14,15]$. With improved genetic analysis techniques, in particular genome-wide association studies, genes can now be identified that are likely to contribute to the development of hypertension within populations [16]. However, most genetic mutations have been identified in the nuclear genome [17]; only a few studies have focused on the investigation of the mitochondrial genome in the development of hypertension in populations. Therefore, it is obvious that understanding mtDNA sequence alteration involvement in MIEH may improve understanding of the genetic basis and pathogenesis of MIEH.

MtDNA mutations mainly distributed in the 79088816 region as described previously [18]. In this study, to understand more about the molecular mechanism underlying $\mathrm{MIEH}$, we undertook screening of study in the mtDNA 7908-8816 region in hypertensive and normotensive subjects in a systematic and extensive manner. We explored inherited and clinical evidence to observe the relationship between the mitochondrial genome and MIEH. We decided to focus upon a Chinese Han population, as the morbidity of $\mathrm{EH}$ in Chinese adults is nearly $11.8 \%$ [19] but there is a limited amount of study on this racial group.

\section{Methods \\ Subjects}

This was a case control study of 300 unrelated patients with MIEH and 300 healthy control subjects. The MIEH participants were selected according to the following inclusion criteria: (1) outpatients or in-patients underwent a regular medical check-up at the Department of Cardiology in Northern Jiangsu People's Hospital from June 2009 to June 2015; (2) more than 18 years old; (3) with a diagnosis of primary hypertension; (4) not receiving antihypertensive medication; (5) diagnosed with $\mathrm{MIEH}$ according to the maternal transmission of EH within generations, which was transmitted by the mother or her relatives and not by the father. Patients were excluded if they were diagnosed with: (1) secondary hypertension (for example renal arterial sterosis, hyperaldosteronism, aortic coarctation, and pheochromocytoma); (2) congenital heart diseases; and (3) presence of organic valve diseases.

Three hundred gender-matched healthy subjects were also selected as the control group. Controls were healthy Yangzhou residents who accepted annual examination in the physical examination center of Northern Jiangsu People's Hospital from June 2009 to June 2015. They were chosen randomly from the daily appointment list and were gender matched with the MIEH group. The inclusion criteria for the control subjects were: (1) no personal or family history of hypertension, and (2) a systolic blood pressure (SBP) of $<130 \mathrm{mmHg}$ and a diastolic blood pressure (DBP) of $<85 \mathrm{mmHg}$. The occurrence of hypertension in one or both biologic parents was considered to be a positive family history of essential hypertension. All study participants were interviewed and then evaluated to identify both personal and medical histories of clinical abnormalities.

Verbal Informed consent, medical history, clinical evaluations and genetic analysis were obtained from all participants involved in the study. The reason for receiving verbal consent is that genetic analysis is used for diagnosis, not for treatment. There was no harm to the patients. The protocol was conducted in accordance with the Helsinki declaration and approved by the ethics committee of the Northern Jiangsu People's Hospital.

\section{Data collection}

Height and weight were both measured when the subjects had fasted overnight and were wearing only underwear. Body mass index (BMI) was calculated as weight in kilograms divided by height in squared meters $\left(\mathrm{kg} / \mathrm{m}^{2}\right)$. Blood pressure was measured by an experienced physician using a mercury column sphygmomanometer according to the World Health Organization (WHO) standardized criteria [20]. The physician was blinded to the study information of the subjects. Systolic and diastolic blood pressure were indicated by the first 
and fifth Korotkoff sounds, respectively. Three systolic and diastolic blood pressure readings were taken and the mean was used as the blood pressure measurement. The hypertension was defined according to the 2010 Chinese guidelines for the management of hypertension [21]: under the condition of no antihypertensive drugs treatment, the systolic blood pressure is higher than $140 \mathrm{mmHg}$ and/or diastolic blood pressure is higher than $90 \mathrm{mmHg}$ measured three times on different days. After 12-h fast, $4 \mathrm{ml}$ venous blood were drawn from the antecubital vein for the measurement of fasting blood glucose (FBG), total cholesterol (TC), low-density lipoprotein cholesterol (LDL), triglycerides (TG), uric acid (UA), creatinine (CR) and blood urea nitrogen (BUN) by an automatic biochemistry analyzer (Hitach 7600DDP, Japan), using Roche biochemical reaction kits.

\section{Mitochondrial DNA analysis}

Genomic DNA was extracted from peripheral blood using standard protocols [22]. DNA was isolated using Promega Wizard Genomic DNA Purification Kit (Madison, WI, USA). Locations considered the main areas for cardiovascular disease as described previously [12] were screened using oligodeoxynucleotides at 7908-8816 bp. Polymerase chain reaction (PCR) was carried out to amplify mitochondrial tRNA ${ }^{\text {Lys }}$ gene using the following primers: forward: 5'-ACGAGTACACCGACTACGG C-3' and reverse: 5' $^{\prime}$ - TGGGTGGTTGGTGTAAATG $\mathrm{A}-3^{\prime}$. PCR was performed in $30 \mu \mathrm{l}$ of the reaction mixture, containing $5.2 \mu \mathrm{l}$ of PCR Master Mix (Qiagen; Hilden, Germany), $2.5 \mu \mathrm{l}$ of each primer, $1 \mu \mathrm{l}$ DNA sample, and $18.8 \mu \mathrm{l}$ of water. The cycling program for PCR consisted of one cycle of $95^{\circ} \mathrm{C}$ for 5 min and then 35 cycles of $95^{\circ} \mathrm{C}$ for $30 \mathrm{~s}, 54{ }^{\circ} \mathrm{C}$ for $30 \mathrm{~s}$ and $72{ }^{\circ} \mathrm{C}$ for $60 \mathrm{~s}$ with a full extension cycle of $72{ }^{\circ} \mathrm{C}$ for $10 \mathrm{~min}$ in a 9700 Thermocycler (Perkin-Elmer Applied Biosystems, Norwalk, USA). Each fragment was purified and subsequently analyzed by direct sequencing with $\mathrm{ABI}$ 3730 Sequence Analysis software (Applied Biosystems, Inc., Foster City, CA, USA) using the BigDye Terminator v1.1 kit (ABI Company, Carlsbad, CA, USA), and SeqWeb program GAP(GCG) was used for analysis referring to the updated consensus Cambridge sequence [23]. Pathogenic variants were identified from MitoMap (http://www.mitomap.org/) [24].

\section{Statistical analysis}

Statistical analysis was performed using $\mathrm{R}$ and SPSS software (version 16.0; SPSS Inc., Chicago, IL, USA). For comparison of the MIEH group and control group, continuous variables were first tested for normal distribution by Kolmogorov-Smirnov test and then presented in terms of mean \pm standard deviation (SD). Discrete variables in the groups were expressed as frequency. Student's $t$ test and Fisher's exact $\mathrm{t}$ test were used to identify the associations between potential continuous and discrete factors and MIEH respectively. A multiple testing adjusted $P$-value of $<0.05$ was considered as statistically significant.

\section{Results}

\section{Clinical evaluation of baseline characteristics}

The general data of study participants is summarized in Table 1. In this study, no significant differences were found in age, gender, or total cholesterol between the two groups. There was a statistical difference for BMI, waist circumference (WC), abdominal circumference (AC), TG, LDL, FBG, UA, CR and BUN $(P<0.05)$ between the two groups.

\section{mtDNA analysis}

Data comparing the frequency of mtDNA variants in the 300 balanced cases and controls are presented in Table 2 . The distribution of the number of observed mutations in mtDNA 7908 8816 bp for all the participants is shown in Fig. 1. As shown in Table 3, we found a total of 40 mutation sites in the $300 \mathrm{MIEH}$ subjects from the mutation analysis (Additional file 1). The mutations were mainly distributed in the regions of the ATP6 binding site, and the site of highest mutation frequency was m. 8414C > T (Fig. 2).

Table 1 Comparison of baseline clinical data between the MIEH and control groups

\begin{tabular}{|c|c|c|c|}
\hline Subjects & MIEH group & Control group & $p$-value \\
\hline Gender(M/F) & $300(148 / 152)$ & $300(145 / 155)$ & 0.870 \\
\hline Age at test(years) & $66.85 \pm 7.24$ & $65.37 \pm 6.76$ & $0.01^{*}$ \\
\hline Age at onset (years) & $47.46 \pm 6.8$ & NA & \\
\hline $\mathrm{SBP}(\mathrm{mmHg})$ & $148.5 \pm 19.8$ & $145.6 \pm 18.6$ & 0.065 \\
\hline $\mathrm{DBP}(\mathrm{mmHg})$ & $94.8 \pm 8.9$ & $88.4 \pm 12.5$ & $<0.001^{*}$ \\
\hline $\mathrm{BMI}\left(\mathrm{kg} / \mathrm{m}^{2}\right)$ & $25.89 \pm 2.61$ & $23.55 \pm 3.04$ & $<0.001^{*}$ \\
\hline WC $(\mathrm{cm})$ & $87.30 \pm 10.78$ & $78.08 \pm 8.72$ & $<0.001^{*}$ \\
\hline $\mathrm{AC}(\mathrm{cm})$ & $89.51 \pm 10.15$ & $80.98 \pm 7.89$ & $<0.001^{*}$ \\
\hline Alcohol, n (\%) & $72(24)$ & $30(10)$ & $<0.001^{*}$ \\
\hline Current Smoking, n & $66(22)$ & $39(13)$ & $0.005^{*}$ \\
\hline $\mathrm{TG}(\mathrm{mmol} / \mathrm{L})$ & $1.87 \pm 1.22$ & $1.38 \pm 0.81$ & $<0.001^{*}$ \\
\hline $\mathrm{TC}(\mathrm{mmol} / \mathrm{L})$ & $4.59 \pm 1.90$ & $4.29 \pm 1.18$ & $0.021^{*}$ \\
\hline $\mathrm{LDL}(\mathrm{mmol} / \mathrm{L})$ & $2.64 \pm 1.02$ & $2.03 \pm 1.35$ & $<0.001^{*}$ \\
\hline FBG (mmol/L) & $5.18 \pm 2.19$ & $4.35 \pm 0.84$ & $<0.001^{*}$ \\
\hline UA(umol/L) & $367.00 \pm 127.27$ & $320.38 \pm 78.91$ & $<0.001^{*}$ \\
\hline $\mathrm{Cr}(\mathrm{ummol} / \mathrm{L})$ & $103.36 \pm 33.71$ & $86.38 \pm 30.71$ & $<0.001^{*}$ \\
\hline BUN(mmol/L) & $5.75 \pm 2.04$ & $4.87 \pm 1.78$ & $<0.001^{*}$ \\
\hline
\end{tabular}

Abbreviations: $F$ female, $M$ male, SBP Systolic blood pressure, DBP Diastolic blood pressure, $B M I$ Body mass index, WC waist circumference, $A C$ abdomen circumference, $T G$ triglyceride, $T C$ total cholesterol, $L D L$ low-density lipoprotein cholesterol, FBG fasting blood glucose, UA uric acid, $\mathrm{Cr}$ creatinine, BUN blood urea nitrogen

*: A $P$ value $<0.05$ was marked by a star 
Table 2 Distribution of mtDNA sequence analyses at positions 7908-8816

\begin{tabular}{llllll}
\hline Gene & Position & Length & Control group $(\mathrm{n}(\%))$ & MIEH group $(\mathrm{n}(\%))$ & Fisher's exact $P$ value \\
\hline COXII & $7908-8269$ & 684 & $7(2.3 \%)$ & $28(9.3 \%)$ & $<0.001$ \\
tRNA $^{\text {Lys }}$ & $8295-8364$ & 70 & 0 & $4(1.3 \%)$ & 0.124 \\
ATP8 & $8366-8572$ & 207 & $11(3.7 \%)$ & $90(30 \%)$ & $<0.001$ \\
ATP6 & $8527-8816$ & 290 & $14(4.7 \%)$ & $115(38 \%)$ & $<0.001$ \\
\hline
\end{tabular}

These results all showed that the MIEH group had more mtDNA variations in frequency and density than the control group. Three SNPs were significantly $(P<0.001)$ different between the MIEH and the control groups: C8414T (leucine to phenylalanine, belongs to haplogroup D) in ATP8 gene, A8701G in ATP6 gene (threonine to alanine, belongs haplogroup $\mathrm{M}$ ), and G8584A in ATP6 gene (alanine to threonine) (Figs. 3 and 4).

Forty different heteroplasmic or homoplasmic mutations were simultaneously identifed in 4 genes: COXII, tRNA ${ }^{\text {Lys }}$, ATP8 and ATP 6 gene. We found a total of 38 homoplasmic mutations in $182 \mathrm{MIEH}$ subjects. Two heteroplasmic mutations of $\mathrm{m} .8563 \mathrm{~A}>\mathrm{T}$ and $\mathrm{m} .8031 \mathrm{C}>$ A were found in $2 \mathrm{MIEH}$ subjects. The MIEH subjects harbored more variants $(P<0.01)$ than the controls with respect to the amino-acid changes and coding sequence variants. Among the MIEH individuals, an intriguing observation was that there were m.8273_8281del mutations in 59 MIEH group patients (Fig. 5). These observations suggested a positive correlation between mtDNA mutation and MIEH.

\section{Discussion}

This study aimed to investigate mtDNA 7908-8816 region mutations in a Chinese population of patients with MIEH. The results showed that the patients in the
MIEH group harbored more mtDNA variants than the control group. We simultaneously identified 40 different heteroplasmic or homoplasmic mutations in 4 genes: COXII, tRNA ${ }^{\text {Lys }}$, ATP8 and ATP6. As previously reported, most pathogenic mtDNA mutations are in tRNAs [25]. Mutations in protein-encoding genes are frequently associated with ATPase dysfunction [26]. Failure in tRNA metabolism will lead to the deficiency of mitochondrial protein synthesis [27]. Defects in mitochondrial translation consequently result in a respiratory phenotype and a decrease in ATP production,may reduce the production of ROS, and subsequently have the potential role to affect the course of hypertension [28]. The mtDNA variations were mainly distributed in regions of ATP6 binding sites, and the site of highest mutation frequency was $\mathrm{m} .8414 \mathrm{C}>\mathrm{T}$. Three single base pair changes were significantly different between the MIEH and control groups, namely C8414T in ATP8, and A8701G and G8584A in ATP6. It is becoming established that mitochondrial damage and dysfunction are important factors in cardiovascular disease [29]. To our best knowledge, this is one of the first large-scale population-based systematic screens for mitochondrial mutations and their effects on MIEH in the Chinese population. Systematic study of the relationship between disease and mtDNA mutation is important to assist with

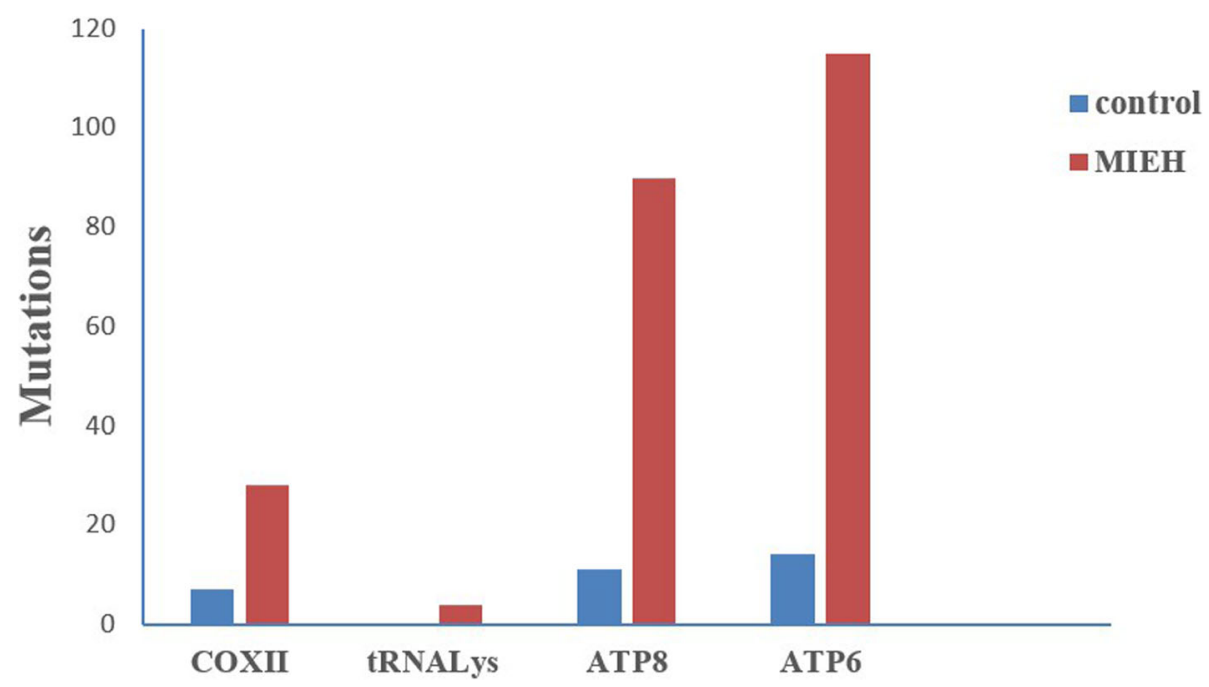

Fig. 1 Distribution histogram of the mtDNA sequences in 7908 8816 
Table 3 Mutation sites of mtDNA in MIEH individuals and controls

\begin{tabular}{|c|c|c|c|c|c|c|c|c|}
\hline \multirow{2}{*}{$\begin{array}{l}\text { Site of } \\
\text { mutation }\end{array}$} & \multirow[t]{2}{*}{ Gene } & \multirow[t]{2}{*}{ Replacement } & \multicolumn{2}{|c|}{ Number of mutations(n) } & \multirow{2}{*}{$\begin{array}{l}\text { Fisher's exact } \\
P \text { value }\end{array}$} & \multirow{2}{*}{$\begin{array}{l}\text { Conservation } \\
(H / B / M / X)^{a}\end{array}$} & \multirow{2}{*}{$\begin{array}{l}\text { Previously } \\
\text { reported }^{b}\end{array}$} & \multirow[t]{2}{*}{ Change of Amino acid } \\
\hline & & & (MIEH) & (Controls) & & & & \\
\hline 8020 & COXII & $\mathrm{G}$ to $\mathrm{A}$ & 1 & 0 & 1 & $\mathrm{G} / \mathrm{T} / \mathrm{C} / \mathrm{G}$ & Yes & non-synonymous variant \\
\hline 8027 & COXII & $\mathrm{G}$ to $\mathrm{A}$ & 4 & 2 & 0.686 & $\mathrm{G} / \mathrm{C} / \mathrm{C} / \mathrm{A}$ & Yes & non-synonymous variant \\
\hline 8078 & COXII & $\mathrm{G}$ to $\mathrm{A}$ & 2 & 1 & 1 & $\mathrm{G} / \mathrm{G} / \mathrm{A} / \mathrm{C}$ & Yes & non-synonymous variant \\
\hline 8149 & COXII & A to $G$ & 3 & 1 & 0.624 & $\mathrm{~A} / \mathrm{A} / \mathrm{C} / \mathrm{T}$ & Yes & non-synonymous variant \\
\hline 8152 & COXII & $\mathrm{G}$ to $\mathrm{A}$ & 2 & 0 & 0.499 & $\mathrm{G} / \mathrm{A} / \mathrm{C} / \mathrm{C}$ & Yes & non-synonymous variant \\
\hline 8176 & COXII & T to $C$ & 2 & 0 & 0.499 & $\mathrm{~T} / \mathrm{A} / \mathrm{C} / \mathrm{T}$ & Yes & non-synonymous variant \\
\hline 8200 & COXII & T to $C$ & 5 & 1 & 0.216 & $\mathrm{~T} / \mathrm{T} / \mathrm{T} / \mathrm{C}$ & Yes & non-synonymous variant \\
\hline 8251 & COXII & $\mathrm{G}$ to $\mathrm{A}$ & 6 & 1 & 0.123 & $\mathrm{G} / \mathrm{T} / \mathrm{T} / \mathrm{T}$ & Yes & non-synonymous variant \\
\hline 8269 & COXII & $\mathrm{G}$ to $\mathrm{A}$ & 2 & 1 & 1 & $\mathrm{G} / \mathrm{A} / \mathrm{A} / \mathrm{C}$ & Yes & non-synonymous variant \\
\hline 8348 & tRNA ${ }^{\text {Lys }}$ & A to $G$ & 2 & 0 & 0.499 & $\mathrm{~A} / \mathrm{T} / \mathrm{T} / \mathrm{G}$ & Yes & non-synonymous variant \\
\hline 8380 & ATP8 & T to $C$ & 1 & 0 & 1 & $\mathrm{~T} / \mathrm{T} / \mathrm{C} / \mathrm{C}$ & No & non-synonymous variant \\
\hline 8392 & ATP8 & $\mathrm{G}$ to $\mathrm{A}$ & 2 & 0 & 0.499 & $\mathrm{G} / \mathrm{A} / \mathrm{T} / \mathrm{T}$ & Yes & non-synonymous variant \\
\hline 8414 & ATP8 & $C$ to $T$ & 60 & 7 & $9.883 e-13$ & $\mathrm{C} / \mathrm{T} / \mathrm{A} / \mathrm{T}$ & Yes & non-synonymous variant \\
\hline 8440 & ATP8 & A to $G$ & 1 & 0 & 1 & $\mathrm{~A} / \mathrm{C} / \mathrm{C} / \mathrm{T}$ & Yes & non-synonymous variant \\
\hline 8452 & ATP8 & A to $G$ & 1 & 0 & 1 & $\mathrm{~A} / \mathrm{A} / \mathrm{G} / \mathrm{T}$ & No & non-synonymous variant \\
\hline 8459 & ATP8 & A to $G$ & 4 & 1 & 0.373 & $\mathrm{~A} / \mathrm{T} / \mathrm{C} / \mathrm{G}$ & No & non-synonymous variant \\
\hline 8467 & ATP8 & $C$ to $T$ & 2 & 0 & 0.499 & C/A/T/T & No & non-synonymous variant \\
\hline 8470 & ATP8 & A to $G$ & 2 & 0 & 0.499 & $\mathrm{~A} / \mathrm{C} / \mathrm{A} / \mathrm{T}$ & Yes & non-synonymous variant \\
\hline 8473 & ATP8 & T to $C$ & 4 & 1 & 0.373 & $\mathrm{~T} / \mathrm{A} / \mathrm{A} / \mathrm{C}$ & Yes & non-synonymous variant \\
\hline 8557 & ATP8 & $\mathrm{G}$ to $\mathrm{A}$ & 1 & 0 & 1 & $\mathrm{G} / \mathrm{C} / \mathrm{G} / \mathrm{T}$ & Yes & non-synonymous variant \\
\hline 8563 & ATP8 & A to $G$ & 4 & 1 & 0.373 & $\mathrm{~A} / \mathrm{T} / \mathrm{G} / \mathrm{C}$ & Yes & non-synonymous variant \\
\hline 8563 & ATP8 & A to $T$ & 1 & 0 & 1 & $\mathrm{~A} / \mathrm{T} / \mathrm{G} / \mathrm{C}$ & No & non-synonymous variant \\
\hline 8584 & ATP6 & $\mathrm{G}$ to $\mathrm{A}$ & 46 & 5 & $5.188 \mathrm{e}-10$ & $\mathrm{G} / \mathrm{T} / \mathrm{C} / \mathrm{A}$ & Yes & non-synonymous variant \\
\hline 8593 & ATP6 & A to $G$ & 1 & 0 & 1 & $\mathrm{~A} / \mathrm{C} / \mathrm{C} / \mathrm{A}$ & No & non-synonymous variant \\
\hline 8654 & ATP6 & T to $C$ & 1 & 0 & 1 & $\mathrm{~T} / \mathrm{A} / \mathrm{A} / \mathrm{G}$ & Yes & non-synonymous variant \\
\hline 8656 & ATP6 & A to $G$ & 3 & 1 & 0.624 & $\mathrm{~A} / \mathrm{G} / \mathrm{C} / \mathrm{T}$ & No & non-synonymous variant \\
\hline 8684 & ATP6 & $\mathrm{C}$ to $\mathrm{T}$ & 9 & 2 & 0.063 & $\mathrm{C} / \mathrm{G} / \mathrm{T} / \mathrm{T}$ & Yes & non-synonymous variant \\
\hline 8701 & ATP6 & A to $G$ & 49 & 6 & $3.445 e-10$ & $\mathrm{~A} / \mathrm{A} / \mathrm{T} / \mathrm{A}$ & Yes & non-synonymous variant \\
\hline 8723 & ATP6 & $\mathrm{G}$ to $\mathrm{A}$ & 1 & 0 & 1 & $\mathrm{G} / \mathrm{A} / \mathrm{A} / \mathrm{T}$ & No & non-synonymous variant \\
\hline 8190 & COXII & $\mathrm{C}$ to $\mathrm{T}$ & 1 & 0 & 1 & $\mathrm{C} / \mathrm{T} / \mathrm{A} / \mathrm{A}$ & No & synonymous variant \\
\hline 8343 & tRNA $A^{\text {Lys }}$ & A to $G$ & 2 & 0 & 0.499 & $\mathrm{~A} / \mathrm{T} / \mathrm{A} / \mathrm{C}$ & Yes & synonymous variant \\
\hline 8403 & ATP8 & T to $C$ & 1 & 0 & 1 & $\mathrm{~T} / \mathrm{A} / \mathrm{G} / \mathrm{C}$ & No & synonymous variant \\
\hline 8409 & ATP8 & $C$ to $T$ & 3 & 1 & 0.624 & $\mathrm{C} / \mathrm{T} / \mathrm{A} / \mathrm{T}$ & No & synonymous variant \\
\hline 8448 & ATP8 & T to $C$ & 2 & 0 & 0.499 & $\mathrm{~T} / \mathrm{A} / \mathrm{C} / \mathrm{G}$ & Yes & synonymous variant \\
\hline 8503 & ATP8 & T to $C$ & 1 & 0 & 1 & $\mathrm{~T} / \mathrm{T} / \mathrm{T} / \mathrm{T}$ & Yes & synonymous variant \\
\hline 8604 & ATP6 & T to $C$ & 1 & 0 & 1 & $\mathrm{~T} / \mathrm{C} / \mathrm{A} / \mathrm{A}$ & Yes & synonymous variant \\
\hline 8614 & ATP6 & T to $C$ & 1 & 0 & 1 & T/T/A/T & Yes & synonymous variant \\
\hline 8643 & ATP6 & $C$ to $T$ & 1 & 0 & 1 & $\mathrm{C} / \mathrm{C} / \mathrm{C} / \mathrm{T}$ & No & synonymous variant \\
\hline 8745 & ATP6 & A to $G$ & 1 & 0 & 1 & $\mathrm{~A} / \mathrm{A} / \mathrm{C} / \mathrm{G}$ & No & synonymous variant \\
\hline 8749 & ATP6 & T to $C$ & 1 & 0 & 1 & $\mathrm{~T} / \mathrm{A} / \mathrm{T} / \mathrm{T}$ & Yes & synonymous variant \\
\hline
\end{tabular}

${ }^{\mathrm{a}} \mathrm{H} / \mathrm{B} / \mathrm{M} / \mathrm{X}$ means human/bovine/mouse/xenopus

${ }^{\mathrm{b}} \mathrm{See}$ http//www.mitomap.org and http://www.genpat.uu.se/mtDB/. Previously reported means the variant was ever reported in a database 


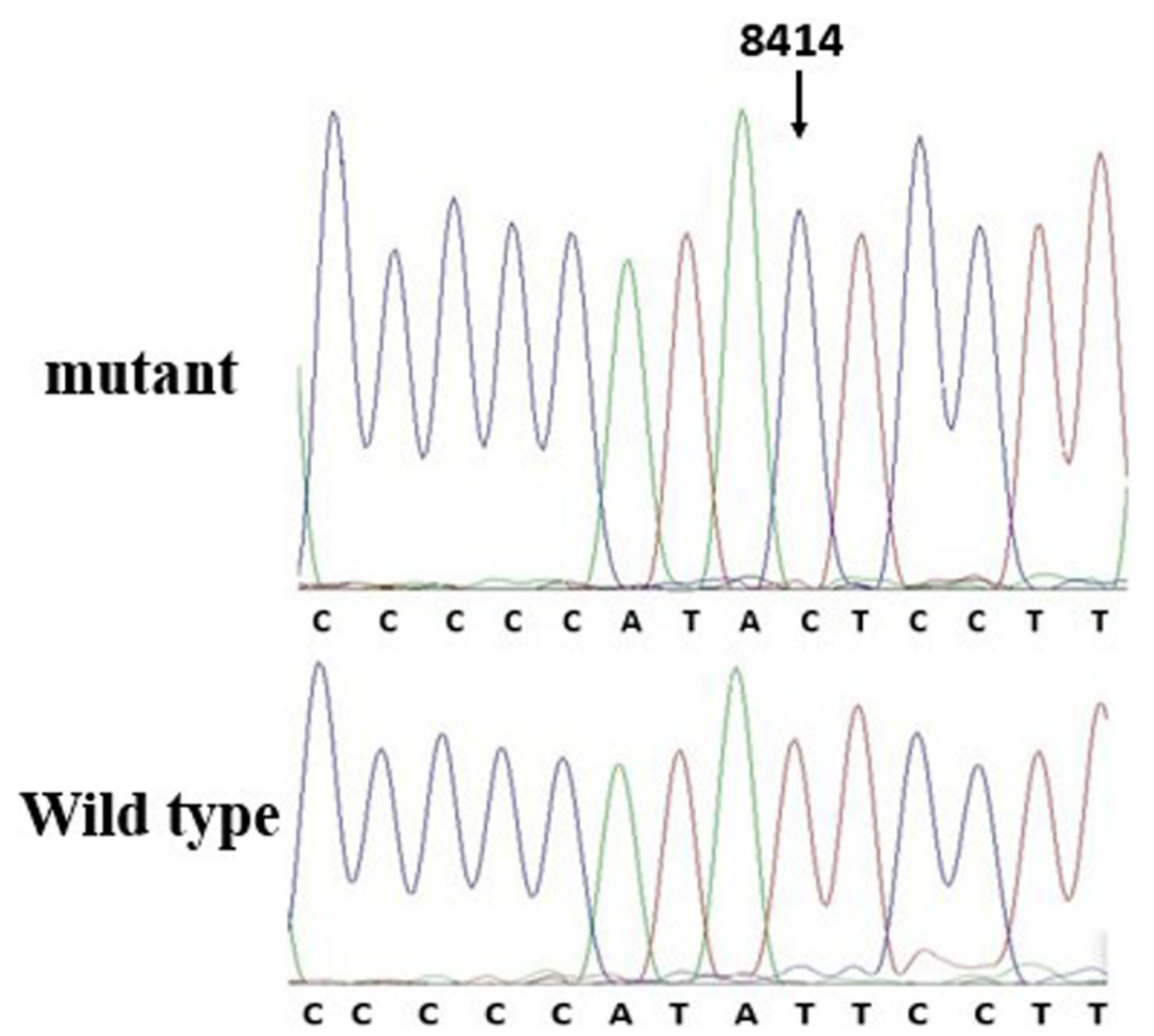

Fig. $\mathbf{2}$ Identification of the m.8414C > T mutation in the mitochondrial ATP8 gene. Arrow indicates the position of the ATP8 gene mutation

our understanding of the mechanism of the mutation and its relationship to disease, but is also able to improve diagnosis, prevention and treatment of $\mathrm{EH}$.

Blood glucose, blood lipids, creatinine, urea nitrogen and other biochemical abnormalities are all very closely related to the chance of developing primary hypertension [30]. In order to clarify whether mtDNA affects the biochemical indicators of the MIEH individuals, we compared and analyzed the biochemical abnormalities of the MIEH and normal individuals. Clinical examination and evaluation of all available members in this study suggested that MIEH subjects presented significantly higher values than those of non-maternal members in BMI, WC, AC, TG, LDL, FBG, UA, CR and BUN. In our study, participants with MIEH were overweight or obese compared to participants with normal blood pressure. A normal body weight (BMI $18.5-24.9 \mathrm{~kg} / \mathrm{m}^{2}$ ) should be maintained for prevention and management of hypertension [31]. The occurrence and development of MIEH, can involve these factors or these factors might occur as a result of the development of MIEH, which leads to the damage or deterioration of the target organ.

Many studies, including the analysis of maternally transmitted hypertension in a large Han Chinese pedigree, have acknowledged the role of inherited mtDNA mutations in familial MIEH [32, 33]. Here, we undertook mutational analysis of the mitochondrial DNA 79088816 region using PCR amplification and then sequence analysis of the PCR fragments. The present experiment showed that there were more mtDNA variations in frequency and density in the MIEH patients than those who were normotensives (NT). Among these mutations, ATP6 is a hotspot for pathogenic mutations associated with MIEH. The occurrence of mtDNA mutations in these genetically unrelated subjects affected by MIEH suggests that mutations may participate in key functional development processes of EH. There are hundreds of mitochondria and thousands of mtDNAs in a mammalian cell and the close proximity of mtDNA within mitochondria with ROS generation sites means that mtDNA is vulnerable to a high level of mutation without an efficient DNA protection and repair system [18].

Mutations of mtDNA may lead to disease, and the significant determinant of their clinical outcomes are likely to correlate with the amount of mutated mtDNA [34]. Some MIEH patients in the study were part of one family branch. Among the MIEH individuals, there was a high mutation frequency and density in mtDNA m.8584G $>\mathrm{A}$ and m.8701A $>$ G mutations. Specifically, an amino acid change at the $\mathrm{m} .8414 \mathrm{C}>\mathrm{T}$ in the ATP8 


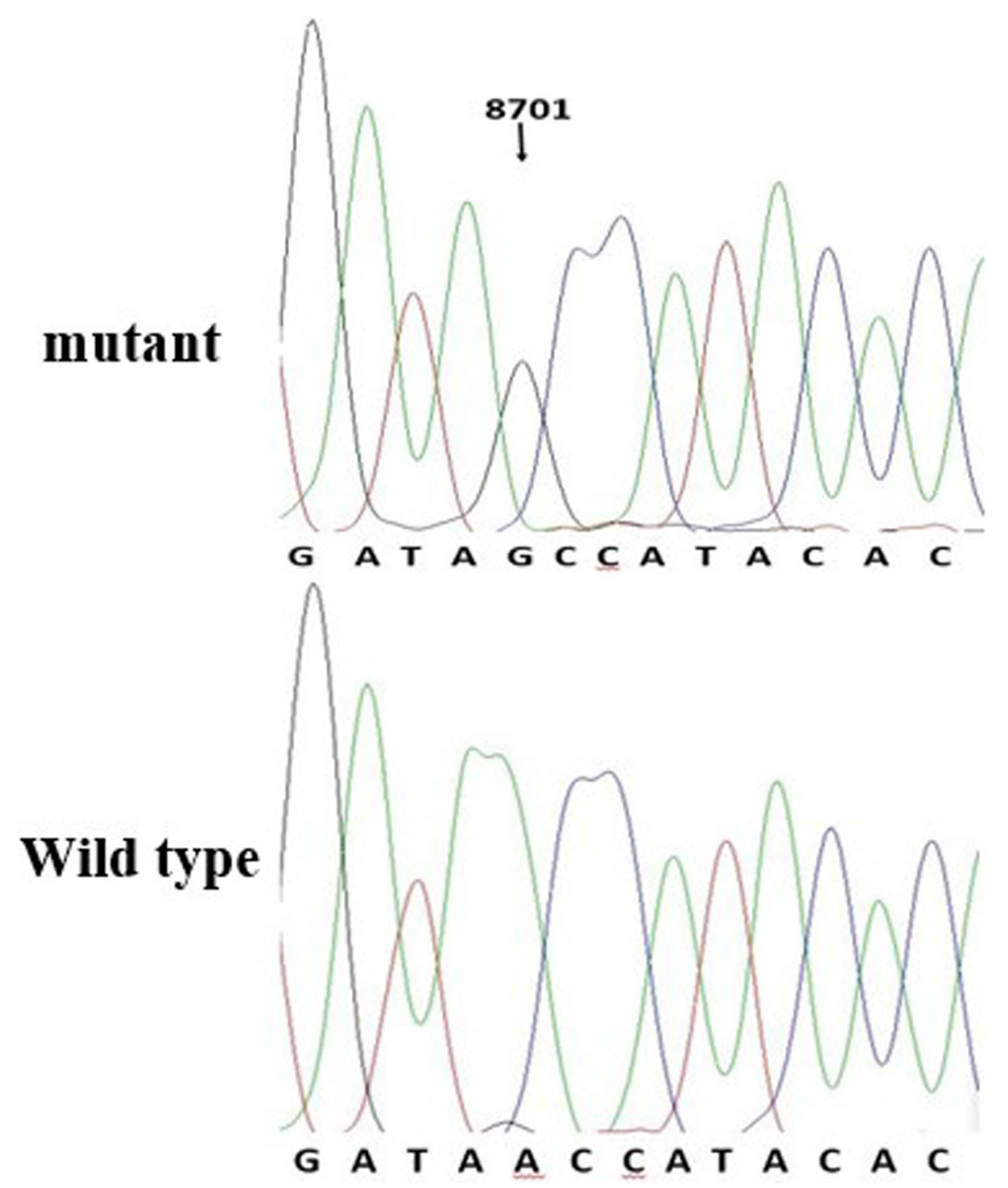

Fig. $\mathbf{3}$ Identification of the m.8701A > G mutation in the mitochondrial ATP6 gene. Arrow indicates the position of the ATP6 gene mutation

gene (leucine to phenylalanine) shows that mtDNA variants may be able to affect the development of hypertension in China [35]. Ancestral variants of mtDNA define population-specific mtDNA lineages or haplogroups. These were first used to trace the origins of different races and allow reconstruction of the migration of humans throughout our ancient history [36]. MtDNA lineages have been shown recently to be more prone to certain disease symptoms, including type-2 diabetes, obesity and atherothrombotic cerebral infarction. Haplogroups can also protect against myocardial infarction and increase lifespan.

MtDNA mutations, including point mutations, deletions, and duplications that affect transcription and translation of mtDNA are implicated in most mitochondrial diseases [37]. Our intriguing observation is that there was a m.8273_8281del mutation in $59 \mathrm{MIEH}$ patients. This 9-bp deletion polymorphism is a phylogenetic marker of studies into evolution trends and population migration [38]. Because of its location, this polymorphism could change either downstream or upstream gene expression. Some crucial structural components of the respiratory chain, such as ATP8, ATP6 are located in the downstream of 9-bp deletion polymorphism [39]. This could affect important respiratory chain structural components, including ATP8 and ATP6 that are located downstream of this polymorphism. If these genes are abnormally expressed they could change oxidative phosphorylation and influence oxidative stress levels [40].

Compared to MIEH patients without mtDNA mutations, the onset time of hypertension for patients with 


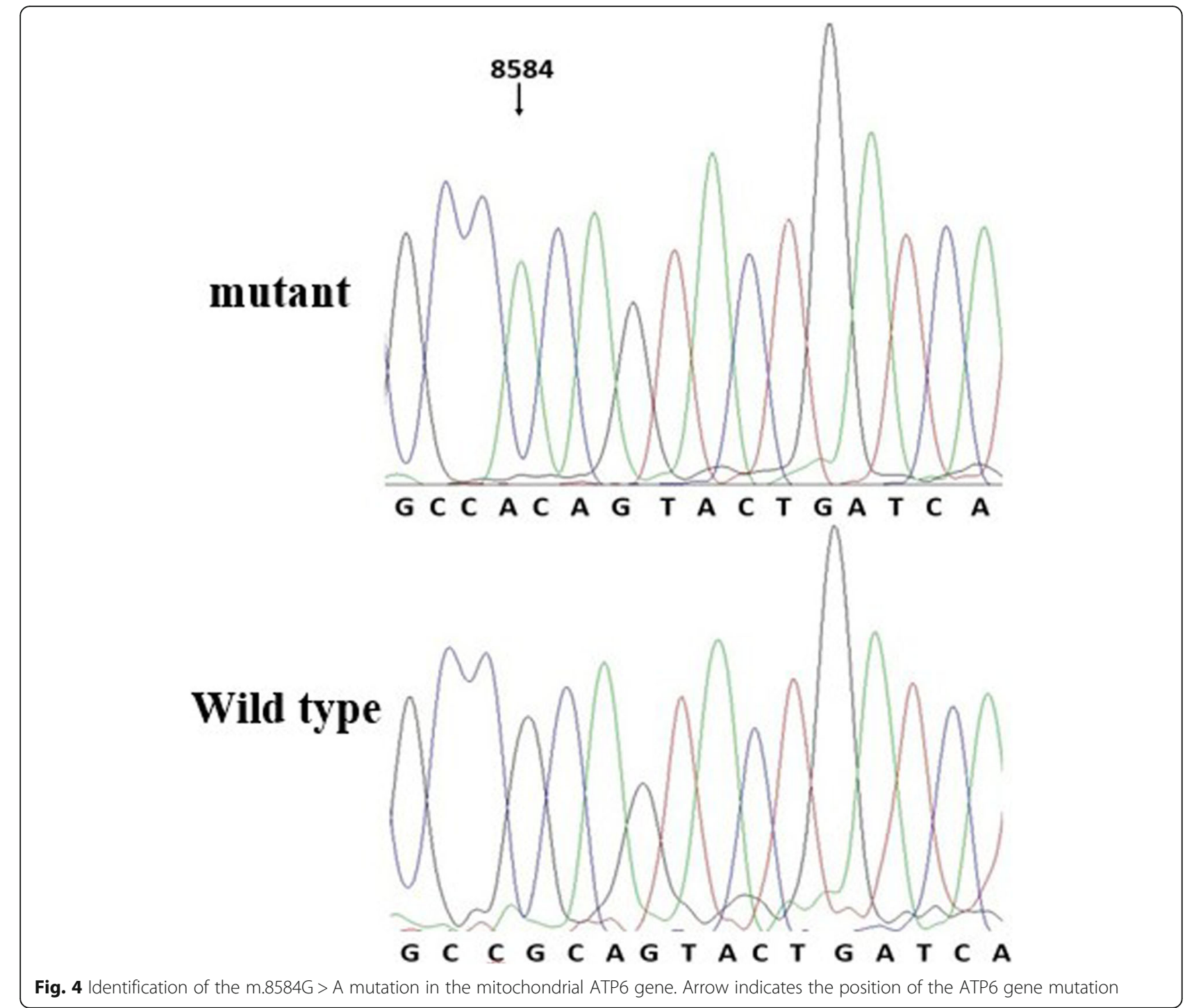

mtDNA mutations was significantly ahead of schedule. This is comparable to the onset time for other Chinese patients with maternally transmitted hypertension [41]. Individuals carrying mtDNA mutations develop hypertension stimulated by environmental factors more easily. Impaired mitochondrial function may contribute increased blood pressure characteristic of aging. Heteroplasmy of mtDNA is strongly associated with hypertension, with the $\mathrm{EH}$ mother carrying the gene mutation, and the children with the same mutation more likely to suffer from hypertension. Regarding amino-acid changes and RNAs variants the MIEH subjects harbored more variants than the controls. A possible reason for mtDNA defects pathogenesis in hypertension is that it decreases energy production, increases ROS production, resulting in oxidative stress, disrupting signal transduction, and leading to cardiovascular and renal damage, ultimately initiating hypertension $[42,43]$. In consist with previous report [44], mtSNPs may affect the development of hypertension in sporadic Chinese hypertensive subjects. Some specific mtSNP within mitochondria may have potential effect in Chinese hypertensives because of their function. How mitochondrial $\mathrm{mtSNPs}$ and/or haplogroups interact synergistically needs to be investigated in further studies. This study was the first step in investigating the role of mitochondria in Chinese hypertensives.

This study has some limitations. One was the small sample size and the single center nature of the study. The conclusions would have been strengthened if the more people had been enrolled. We were not able to control for genetic principal components, which is typically essential in any genomic analysis. Additional limitation was that we were not including people treated with antihypertensives, etc. this would have eliminated quite a large number of people from the analysis. 


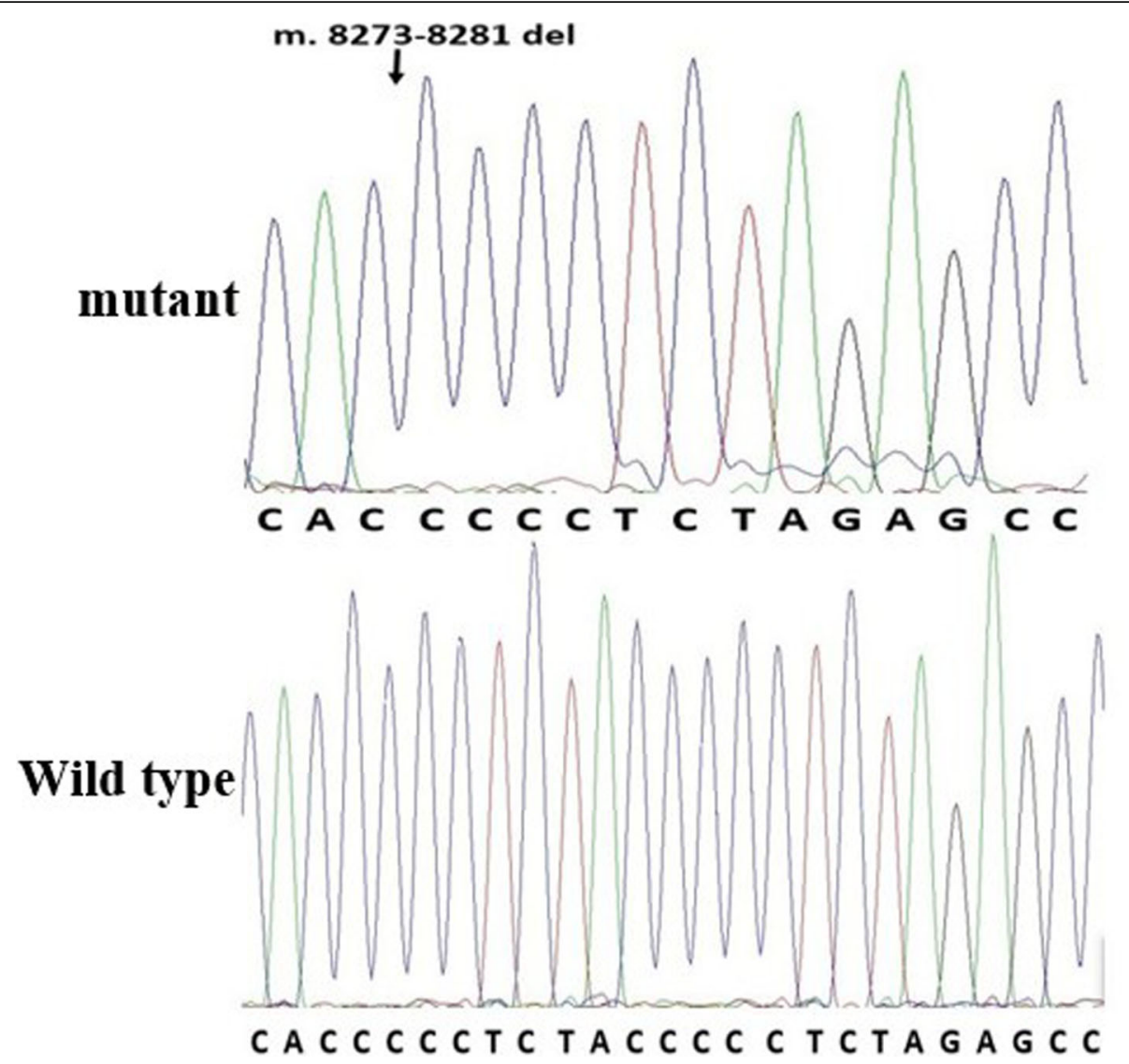

Fig. $\mathbf{5}$ Identification of the m.8273_8281del mutations. Arrow indicates the position of the deletion mutation

\section{Conclusions}

In conclusion, our data convincingly demonstrated the possibility of mitochondrial mutations being involved in the pathological process of MIEH. We also identified mitochondrial genetic characteristics in MIEH individuals. Our findings may be generalizable to other China and Asian populations with a similar lifestyle. The investigation of the role of mitochondrial dysfunction in MIEH provides critical implications on the understanding and treatment of this disorder. The present research serves as a solid foundation for further study on the association between MIEH and mitochondrial dysfunction, and the cause and effect relationship in this population.

\section{Additional file}

Additional file 1: Online mtDNA mutations of the MIEH patients. (RAR $3557 \mathrm{~kb}$ )

\section{Acknowledgements}

We are grateful to Dr. Lei Sun, Dr. Yi Zhang, Dr. Zhengyu Bao and Dr. Jianhua Shen for their valuable advice. We thank the editor and the reviewers whose comments/suggestions helped improve and clarify this manuscript.

\section{Funding}

The design and collection of data of the study was supported by Jiangsu Province Outstanding Medical Talented Leader (JS2006038). The analysis, interpretation of data and in writing the manuscript was supported by Science and Technology Department of Jiangsu Province (No. BL2013022).

\section{Availability of data and materials}

The software of mtDNA mutation analysed during the current study is available at http//www.mitomap.org and http://www.genpat.uu.se/mtDB/. The datasets analysed during the current study is available from the corresponding author on reasonable request.

\section{Authors' contributions}

$Y Z$ and $X G$ carried out the studies, participated in collecting data, and drafted the manuscript. YZ and CX performed the statistical analysis and participated in its design. CX helped to draft the manuscript. All authors read and approved the final manuscript.

Ethics approval and consent to participate

Verbal informed consent was obtained from the subjects involved in the study. The protocol was conducted in accordance with the Declaration of Helsinki and approved by the institutional ethical board of Northern Jiangsu People's Hospital, Jiangsu Province, China.
AC: Abdominal circumference; BMI: Body mass index; DBP: Diastolic blood pressure; EH: Essential hypertension; MIEH: Maternally inherited essential hypertension; MtDNA: Mitochondrial DNA; SBP: Systolic blood pressure; WC: Waist circumference 


\section{Consent for publication}

Not applicable.

\section{Competing interests}

The authors declare that they have no competing interests.

\section{Publisher's Note}

Springer Nature remains neutral with regard to jurisdictional claims in published maps and institutional affiliations.

\section{Author details}

${ }^{1}$ Clinical Medical College, Yangzhou University, Yangzhou 225001, Jiangsu, China. ${ }^{2}$ Department of Cardiology, Northern Jiangsu People's Hospital, Yangzhou 225001, Jiangsu, China. ${ }^{3}$ Department of Biostatistics and Epidemiology, University of Oklahoma Health Science Center, Oklahoma City, OK 73134, USA.

\section{Received: 21 July 2017 Accepted: 25 September 2018 \\ Published online: 16 October 2018}

\section{References}

1. Marian AJ. Mitochondrial genetics and human systemic hypertension. Circ Res. 2011;108(7):784-6

2. Chen BL, Zhang YZ, Luo JQ, Zhang W. Clinical use of azelnidipine in the treatment of hypertension in Chinese patients. Ther Clin Risk Manag. 2015; 11:309-18.

3. Nemati R, Lu J, Ramachandran V, Etemad A, Heidari M, Yahya MJ, et al. Association between the C34T polymorphism of the AMPD1 gene and essential hypertension in Malaysian patients. Genet Mol Res. 2016;15(2):1-11.

4. Guo H, Zhuang XY, Zhang AM, Zhang W, Yuan Y, Guo L, et al. Presence of mutation m.14484T>C in a Chinese family with maternally inherited essential hypertension but no expression of LHON. Biochim Biophys Acta. 2012;1822(10):1535-43

5. Qiu Q, Li R, Jiang P, Xue L, Lu Y, Song Y, et al. Mitochondrial tRNA mutations are associated with maternally inherited hypertension in two Han Chinese pedigrees. Hum Mutat. 2012;33(8):1285-93.

6. Schapira AH. Mitochondrial disease. Lancet. 2006;368(9529):70-82

7. Chen H, Zheng J, Xue L, Meng Y, Wang Y, Zheng B, et al. The 125 rRNA A1555G mutation in the mitochondrial haplogroup D5a is responsible for maternally inherited hypertension and hearing loss in two Chinese pedigrees. Eur J Hum Genet. 2012;20(6):607-12.

8. Liu P, Demple B. DNA repair in mammalian mitochondria: much more than we thought? Environ Mol Mutagen. 2010;51(5):417-26.

9. Lu Z, Chen H, Meng Y, Wang Y, Xue L, Zhi S, et al. The tRNAMet 4435A>G mutation in the mitochondrial haplogroup G2a1 is responsible for maternally inherited hypertension in a Chinese pedigree. Eur J Hum Genet. 2011;19(11):1181-6.

10. Wang S, Li R, Fettermann A, Li Z, Oian Y, Liu Y, et al. Maternally inherited essential hypertension is associated with the novel $4263 \mathrm{~A}>\mathrm{G}$ mutation in the mitochondrial tRNAlle gene in a large Han Chinese family. Circ Res. 2011;108(7):862-70.

11. Muftuoglu M, Mori MP, de Souza-Pinto NC. Formation and repair of oxidative damage in the mitochondrial DNA. Mitochondrion. 2014;17:164-81.

12. Liu Y, Li Y, Gao J, Zhu C, Lan Y, Yang J, et al. Molecular characterization of a Chinese family carrying a novel C4329A mutation in mitochondrial tRNAlle and tRNAGIn genes. BMC Med Genet. 2014;15:84.

13. Jia $Z$, Wang $X$, Qin $Y$, Xue $L$, Jiang $P$, Meng $Y$, et al. Coronary heart disease is associated with a mutation in mitochondrial tRNA. Hum Mol Genet. 2013; 22(20):4064-73.

14. Schon EA, DiMauro S, Hirano M. Human mitochondrial DNA: roles of inherited and somatic mutations. Nat Rev Genet. 2012;13(12):878-90.

15. Liu C, Yang Q, Hwang SJ, Sun F, Johnson AD, Shirihai OS, et al. Association of genetic variation in the mitochondrial genome with blood pressure and metabolic traits. Hypertension. 2012;60(4):949-56.

16. Padmanabhan S, Melander O, Johnson T, Di Blasio AM, Lee WK, Gentilini D, et al. Genome-wide association study of blood pressure extremes identifies variant near UMOD associated with hypertension. PLoS Genet. 2010;6(10): e1001177.

17. Assie G, Letouze E, Fassnacht M, Jouinot A, Luscap W, Barreau O, et al. Integrated genomic characterization of adrenocortical carcinoma. 2014; 46(6):607-12.
18. Zhu HY, Wang SW, Martin LJ, Liu L, Li YH, Chen R, et al. The role of mitochondrial genome in essential hypertension in a Chinese Han population. Eur J Hum Genet. 2009;17(11):1501-6.

19. Teng L, Zheng J, Leng J, Ding Y. Clinical and molecular characterization of a Han Chinese family with high penetrance of essential hypertension. Mitochondrial DNA. 2012;23(6):461-5.

20. Chalmers J, MacMahon S, Mancia G, Whitworth J, Beilin L, Hansson L, et al. World Health Organization-International Society of Hypertension Guidelines for the management of hypertension. Guidelines sub-committee of the World Health Organization. Clin Exp Hypertens. 1999;21(5-6):1009-60.

21. Liu LS. Chinese guidelines for the management of hypertension. Zhonghua Xin Xue Guan Bing Za Zhi 2011. 2010;39(7):579-615.

22. Zhu HY, Wang SW, Liu L, Li YH, Chen R, Wang L, et al. A mitochondrial mutation A4401G is involved in the pathogenesis of left ventricular hypertrophy in Chinese hypertensives. Eur J Hum Genet. 2009;17(2):172-8.

23. Gomez-Carballa A, Cerezo M, Balboa E, Heredia C, Castro-Feijoo L, Rica I, et al. Evolutionary analyses of entire genomes do not support the association of mtDNA mutations with Ras/MAPK pathway syndromes. PLoS One. 2011; 6(4):e18348.

24. Ingman M, Gyllensten U. mtDB: human mitochondrial genome database, a resource for population genetics and medical sciences. Nucleic Acids Res. 2006;34(Database issue):D749-51.

25. Jesina P, Tesarova M, Fornuskova D, Vojtiskova A, Pecina P, Kaplanova V, et al. Diminished synthesis of subunit a (ATP6) and altered function of ATP synthase and cytochrome c oxidase due to the mtDNA 2 bp microdeletion of TA at positions 9205 and 9206. Biochem J. 2004;383(Pt. 3):561-71.

26. Liu Y, Zhu Q, Zhu C, Wang X, Yang J, Yin T, et al. Systematic analysis of the clinical and biochemical characteristics of maternally inherited hypertension in Chinese Han families associated with mitochondrial. BMC Med Genet. 2014;7:73.

27. Zheng P, Li S, Liu C, Zha Z, Wei X, Yuan Y. Mitochondrial tRNA(ala) C5601T mutation may modulate the clinical expression of tRNA(met) A4435G mutation in a Han Chinese family with hypertension. Clin Exp Hypertens. 2018;40(6):595-600.

28. Li H, Geng J, Yu H, Tang X, Yang X, Xue L. Mitochondrial tRNA(Thr) $15909 \mathrm{~A}>\mathrm{G}$ mutation associated with hypertension in a Chinese Han pedigree. Biochem Biophys Res Commun. 2018;495(1):574-81.

29. Samuels DC, Li C, Li B, Song Z, Torstenson E, Boyd Clay H, et al. Recurrent tissue-specific mtDNA mutations are common in humans. PLoS Genet. 2013;9(11):e1003929.

30. Lu Y, Xiao T, Zhang F, Chen Y, Liu Y, Li Y, et al. Effect of mitochondrial tRNA(Lys) mutation on the clinical and biochemical characteristics of Chinese essential hypertensive subjects. Biochem Biophys Res Commun. 2014:454(4):500-4.

31. Chobanian AV, Bakris GL, Black HR, Cushman WC, Green LA, Izzo JL Jr, et al. Seventh report of the joint National Committee on prevention, detection, evaluation, and treatment of high blood pressure. Hypertension. 2003;42(6): 1206-52.

32. Akhmedov AT, Marin-Garcia J. Mitochondrial DNA maintenance: an appraisal. Mol Cell Biochem. 2015;409(1-2):283-305.

33. Chen X, Zhang Y, Xu B, Cai Z, Wang L, Tian J, et al. The mitochondrial calcium uniporter is involved in mitochondrial calcium cycle dysfunction: underlying mechanism of hypertension associated with mitochondrial tRNA(lle) A4263G mutation. Int J Biochem Cell Biol. 2016;78:307-14.

34. Shimizu A, Mito T, Hayashi C, Ogasawara E, Koba R, Negishi I, et al. Transmitochondrial mice as models for primary prevention of diseases caused by mutation in the tRNA(Lys) gene. Proc Natl Acad Sci U S A. 2014;111(8):3104-9.

35. Mohanty K, Dada R, Dada T. Neurodegenerative eye disorders: role of mitochondrial dynamics and genomics. Asia Pac J Ophthalmol (Phila). 2016:5(4):293-9.

36. Kytovuori L, Lipponen J, Rusanen H, Komulainen T, Martikainen MH, Majamaa K. A novel mutation m.8561C>G in MT-ATP6/8 causing a mitochondrial syndrome with ataxia, peripheral neuropathy, diabetes mellitus, and hypergonadotropic hypogonadism. J Neurol. 2016;263(11):2188-95.

37. Shen $L$, Fang $H$, Chen $T$, He J, Zhang $M$, Wei $X$, et al. Evaluating mitochondrial DNA in cancer occurrence and development. Ann N Y Acad Sci. 2010;1201:26-33.

38. Yao YG, Watkins WS, Zhang YP. Evolutionary history of the mtDNA 9-bp deletion in Chinese populations and its relevance to the peopling of east and Southeast Asia. Hum Genet. 2000;107(5):504-12. 
39. Kleidon J, Plesofsky N, Brambl R. Transcripts and transcript-binding proteins in mitochondria of Neurospora crassa. Mitochondrion. 2003;2(5):345-60.

40. Komandur S, Venkatasubramanian S, Alluri RV, Rao P, Rao P, Hasan Q. Mitochondrial insertion-deletion polymorphism: role in disease pathology. Genet Test Mol Biomarkers. 2011;15(5):361-4.

41. Aure K, Dubourg O, Jardel C, Clarysse L, Sternberg D, Fournier E, et al. Episodic weakness due to mitochondrial DNA MT-ATP6/8 mutations. Neurology. 2013;81(21):1810-8.

42. Moskalev AA, Aliper AM, Smit-McBride Z, Buzdin A, Zhavoronkov A. Genetics and epigenetics of aging and longevity. Cell Cycle. 2014;13(7):1063-77.

43. Nazarewicz RR, Dikalov SI. Mitochondrial ROS in the prohypertensive immune response. Am J Physiol Regul Integr Comp Physiol. 2013;305(2):R98-100.

44. Chen J, Zhao X, Wang H, Chen Y, Wang W, Zhou W, et al. Common variants in TGFBR2 and miR-518 genes are associated with hypertension in the Chinese population. Am J Hypertens. 2014;27(10):1268-76.

Ready to submit your research? Choose BMC and benefit from:

- fast, convenient online submission

- thorough peer review by experienced researchers in your field

- rapid publication on acceptance

- support for research data, including large and complex data types

- gold Open Access which fosters wider collaboration and increased citations

- maximum visibility for your research: over $100 \mathrm{M}$ website views per year

At $\mathrm{BMC}$, research is always in progress.

Learn more biomedcentral.com/submissions 